Krebs:

\section{Nebenwirkungen der Behandlung dämpfen - Lebensqualität verbessern}

Die Weltkrebs-Vereinigung UICC, in der auch die Deutsche Krebsgesellschaft und die Krebshilfe Mitglied sind, kämpft nicht nur gegen den Krebs, sondern auch gegen Vorurteile. Eines davon ist, dass man nach einer Krebsdiagnose ohnedies nichts mehr machen könne. Diesen Aberglauben bis zum Jahr 2025 auszutreiben, ist eines der Ziele, das sich die Organisation in ihrer Welt-Krebsdeklaration vorgenommen hat. Denn nichts zu tun, bedeutet häufig einen Verzicht auf Lebensqualität.

Dies zeigen auch eine Reihe eindrucksvoller Studien. Wie Dr. Carlos A. S. Franca, Radioonkologische Abteilung der katholischen Universität von Rio de Janeiro [1], berichtet, sank bei Brustkrebspatientinnen der Selenspiegel auf rund die Hälfte ab: von im Mittel 86,4 $\mu \mathrm{g}$ auf 47,8 $\mu$ g. Die Arbeitsgruppe um Dr. Franca hatte in ihrer Studie von Dezember 2007 bis August 2008 bei 209 Brustkrebspatientinnen vor und nach der Radiotherapie die Selenspiegel gemessen.

Tendenziell ähnliche Ergebnisse zeigt eine Studie [2] aus Deutschland, die den Zusammenhang von niedrigen Selenspiegeln und behandlungstypischer Diarrhöe untersucht. In dieser Studie zeigte sich, dass höhere Selenspiegel die Nebenwirkungen der Strahlentherapie reduzieren. Patientinnen, deren Selenspiegel durch die Gabe von Natriumselenit (in der Studie: selenase ${ }^{\circledR}$ ) angehoben worden war, hatten nach 5-wöchiger Strahlentherapie signifikant weniger unter Diarrhöe ( $\geq$ CTC-Grad 2: 20,5 vs. $44,5 \%$ ) zu leiden als Patientinnen der Kontroll- gruppe, die kein Selen erhielten ( $p=0,04)$. Die 5-Jahres-Überlebensrate der Patientinnen mit Selen-Ergänzung lag bei 92 , in der Kontrollgruppe bei $83 \%(p=0,34)$. Damit konnte erneut belegt werden, dass die zusätzliche Selengabe die Standardtherapie nicht beeinträchtigt. Die Ergebnisse legen nahe, bei Krebspatienten auf eine ausreichende Selenversorgung zu achten

\section{Referenzen \\ 1 Franca C A S et al. Ann Oncol 2011:22:1109-1112. \\ Mücke R et al.: Int J Radiation Oncol 2010;78:828-835.}

Weitere Informationen bei biosyn Arzneimittel GmbH

Presse- und Öffentlichkeitsarbeit

Schorndorfer Strasse 32, 70734 Fellbach presse@biosyn.de

\section{Natürlich und verträglich:}

\section{Naturmedizin für die Seele}

Laut einer aktuellen Studie [1] nutzen $2 / 3$ aller Bundesbürger Naturheilmittel zur Linderung von psychischen und physischen Beschwerden - Tendenz steigend. Die Behandlung durch naturmedizinische Arzneimittel ist bei Ärzten und Patienten gleichermaßen anerkannt. Längst sind naturmedizinische Therapien also keine Ausnahme mehr.

Der Ruf nach natürlicher Medizin ist in den letzten Jahren auf Seiten der Patienten immer lauter geworden: Bereits 2010 nutzten 72\% aller Patienten Naturheilmittel [2]. So setzen Patienten insbesondere bei nervöser Unruhe vermehrt auf Naturheilmittel. Vor die Wahl gestellt, ziehen sogar $80 \%$ aller Patienten diese Therapie chemischen Medikamenten vor [3].
Seit langem hat sich in diesem Indikationsgebiet der Naturmedizin die Passionsblume bewährt. Sie kann Gefühle von Nervosität und innerer Unruhe schnell mildern. Bereits nach 30 Minuten entfaltet der Passionsblumenextrakt seine entspannende Wirkung [4]. Es gibt sogar Hinweise darauf, dass Passionsblumenkraut in der Wirkungsart chemischen Beruhigungsmitteln ähnlich ist, denn beide wirken $u$. a. über den Nervenbotenstoff Gamma-Aminobuttersäure [5].

Patienten wünschen sich neben der Wirksamkeit vor allem eine gute Verträglichkeit der eingenommenen Arzneimittel. Im Unterschied zu vielen chemischen Beruhigungsmitteln ist ein hochkonzentrierter Passionsblumenextrakt (z.B. Pascoflair $^{\circledR} 425 \mathrm{mg}$ ) sehr gut verträglich, auch die Konzentrationsfähigkeit bleibt erhalten. Darüber hinaus gibt es kein bekanntes Abhängigkeitspotential und keine bekannten Wechselwir- kungen. Somit eignen sich die Arzneimittel auch zur Einnahme über einen längeren Zeitraum und für Menschen, die berufstätig sind.

\section{Referenzen}

1 PASCOE-Studie: Naturmedizin 2012 - Ausbildungssituation an deutschen Hochschulen.

2 Allensbacher Berichte: Naturheilmittel - Helfer vo allem bei Erkältungen. 2010; Archiv-Nummer: 10056.

3 PASCOE-Studie 2007: Repräsentative Umfrage über die Einstellung der Bevölkerung zur Naturmedizin in Deutschland.

4 Dimpfel W et al.: Neuroscience \& Medicine 2012; 3:130-140.

5 Appel K et al.: Phytother Res 2010;25:838-843.

Weitere Informationen bei

PASCOE Naturmedizin

35383 Giessen

info@pascoe.de

www.pascoe.de

\title{
Ticker+++ Ticker+++ Ticker+++ Ticker+++ Ticker+++ Ticker+++ Ticker+++
}

DHU. Das DHU Schüßler-Salz Nr. 7 Magnesium phosphoricum D6 gibt es jetzt auch als Sachet. Aufgrund seiner breiten Einsatzmöglichkeit gehört die Nr. 7 zu den bekanntesten und beliebtesten Schüßler-Salzen. Eine besondere Form der Einnahme ist die «Heiße Sieben». Bisher wurden dafür 10 Tabletten in heißem Wasser aufgelöst. Jetzt kann das fertige Pulver aus dem Beutel direkt in das heiße Wasser gegeben werden und löst sich nach wenigen Löffelumdrehungen auf. Die Bestellnummer für die Apotheke lautet: PZN 10048663.

Deutsche Homöopathie-Union

Dr. Wolfgang Kern

www.schuessler.dhu.de
Schwabe. Oft liegt bei Alzheimer-Patienten zusätzlich eine vaskuläre Schädigung vor. Prof. Dr. Serge Gauthier aus Quebec, Kanada, präsentierte auf dem ECNP-Kongress in Barcelona erstmals Daten einer neuen Metaanalyse, die bestätigt: Der Ginkgo-Spezialextrakt EGb $761^{\circledR}$ ist eine evidenzbasierte Therapieoption bei Alzheimer-Demenz mit und ohne zerebrovaskuläre Erkrankung sowie bei vaskulärer Demenz. Patienten mit neuropsychiatrischen Symptomen profitierten besonders.

Dr. Willmar Schwabe GmbH \& Co. KG

Dr. Gunnar P. H. Dietz

wiss-info@schwabe.de
Keinen Grund zur Entwarnung bei der Folatversorgung sieht der Arbeitskreis Folsäure und Gesundheit (AKF). Zwar hat die Deutsche Gesellschaft für Ernährung den empfohlenen Tageswert von 400 auf $300 \mu \mathrm{g}$ gesenkt. Die Lücke zwischen empfohlener und realer Aufnahme bleibt aber groß. Denn nach einer von Wissenschaftlern des Max Rubner-Instituts vorgenommenen Neubewertung ist die Folataufnahme noch geringer als vermutet - bei der Hälfte aller Deutschen sind es weniger als $200 \mu \mathrm{g} / \mathrm{Tag}$.

Arbeitskreis Folsäure \& Gesundheit

info@ak-folsaeure.de

www.ak-folsaeure.de 